\title{
Isolated Attosecond Pulses from Laser-Driven Synchrotron Radiation
}

\author{
J. M. Mikhailova, ${ }^{1,2,3, *}$ M. V. Fedorov, ${ }^{2}$ N. Karpowicz, ${ }^{1}$ P. Gibbon, ${ }^{4}$ V. T. Platonenko, ${ }^{5}$ \\ A. M. Zheltikov, ${ }^{3,5,6}$ and F. Krausz ${ }^{1,7}$ \\ ${ }^{1}$ Max-Planck-Institut für Quantenoptik, Hans-Kopfermann-Strasse 1, 85748 Garching, Germany \\ ${ }^{2}$ A. M. Prokhorov General Physics Institute, Russian Academy of Sciences, Vavilova Street 38, 119991 Moscow, Russia \\ ${ }^{3}$ Russian Quantum Center, Novaya Street 100, 143025 Skolkovo, Moscow Region, Russia \\ ${ }^{4}$ Juelich Supercomputing Centre, Forschungszentrum Juelich, 52425 Juelich, Germany \\ ${ }^{5}$ M. V. Lomonosov Moscow State University, Leninskie Gory 1, 119992 Moscow, Russia \\ ${ }^{6}$ Department of Physics and Astronomy, Texas A\&M University, College Station, Texas 77843-4242, USA \\ ${ }^{7}$ Department für Physik, Ludwig-Maximilians-Universität, Am Coulombwall 1, 85748 Garching, Germany
}

(Received 18 April 2012; published 13 December 2012)

\begin{abstract}
A quantitative theory of attosecond pulse generation in relativistically driven overdense plasma slabs is presented based on an explicit analysis of synchrotron-type electron trajectories. The subcycle, fieldcontrolled release, and subsequent nanometer-scale acceleration of relativistic electron bunches under the combined action of the laser and ionic potentials give rise to coherent radiation with a high-frequency cutoff, intensity, and radiation pattern explained in terms of the basic laws of synchrotron radiation. The emerging radiation is confined to time intervals much shorter than the half-cycle of the driver field. This intuitive approach will be instrumental in analyzing and optimizing few-cycle-laser-driven relativistic sources of intense isolated extreme ultraviolet and x-ray pulses.
\end{abstract}

PACS numbers: 52.38.Ph, 42.65.Ky, 42.65.Re, 52.50.Jm

Progress in ultraintense lasers capable of accelerating electrons to relativistic velocities [1] raises the question of whether laser technologies will ever be able to make the cutting-edge science of large accelerators accessible to a broad range of researchers in moderate-size laser labs. From the radiation physics perspective, an advantage of lasers is their ability to generate unprecedentedly short pulses of extreme ultraviolet (XUV) and x-ray radiation [2-5]. High-order harmonic generation in gas jets [6-8] when driven by waveform-controlled few-cycle light [3] enables the generation of sub-100-as XUV pulses [5]. They have allowed unique time-resolved studies of electron dynamics in gases $[9,10]$ and in the condensed phase [11]. However, the flux and photon energy of attosecond pulses generated with the use of this technology are limited [12]. Recent insight into ultrafast laser-plasma interactions [13-21] offers the potential for much higher yields and photon energies by combining advanced concepts of ultrafast optical science and relativistic plasma physics.

The key physical scenarios of coherent XUV and x-ray emission from overdense laser-produced plasmas are (i) radiation through linear mode conversion of highfrequency plasmons excited in a nanoscale plasma density gradient [22], and (ii) radiation from relativistic electrons coherently moving at surfaces of overdense plasmas. The latter, highly nonlinear phenomenon was predicted by numerical simulations [13-15] and described in terms of the Doppler frequency up-shift of light reflected by oscillating plasma surface or "relativistically oscillating mirror" $[13,15,18]$. Such radiation was experimentally observed as high harmonics by several groups [[19-21].
Formation of attosecond pulses in the relativistic regime has been numerically and analytically studied in [16-18].

We show here that even though the spatial scales of the effects are drastically different, the physics of XUV and $\mathrm{x}$-ray emission by electrons in large-scale synchrotron facilities and electrons undergoing laser acceleration to relativistic velocities on a nanometer scale at the surface of a solid is essentially the same. While the synchrotronlike features in relativistic harmonic spectra have been identified in earlier work $[23,24]$, here we demonstrate that the general scaling laws of synchrotron radiation-i.e., the mathematical expressions of its essential properties such as the intensity and spectral cutoff as a function of electron energy and density of the bunch-fully apply to the radiation emitted by electrons in dense plasmas driven by an intense laser field. Known for more than half a century from astrophysical observations $[25,26]$ and grand accelerator experiments [27], synchrotron radiation physics will be shown to (i) offer a new perspective on the radiation and particle-acceleration physics behind ultraintense laser interactions with matter, and (ii) give important insights into the scenarios of attosecond pulse generation by laseraccelerated electrons in overdense plasma slabs.

Let us consider the interaction of an ultrashort relativistic-intensity laser pulse with a slab of overdense plasma. Before the laser pulse arrival, plasma electrons reside in a strong binding potential from the ions exerting a restoring force when electrons start to move [see Supplemental Material [28] for details]. The motion of electrons in the direction orthogonal to the target surface under the combined action of the Lorentz force and the 
ionic restoring force results in their escape into the vacuum at the front surface of plasmas, where the ionic binding potential gradually vanishes and gives birth to high-order harmonic generation or isolated XUV and x-ray pulses. Here, we demonstrate the basic idea for the simplest, normal incidence geometry with the analysis of oblique incidence, which results in largest amplitudes of electron oscillations in the direction normal to the target surface and in greatest energies of attosecond pulses, left to be carried out in future work.

In this case, the physical picture of attosecond emission suggested by particle-in-cell (PIC) simulations is as follows. Electrons are pushed by the magnetic force oscillating with the frequency $2 \omega_{0}\left(\omega_{0}\right.$ is the laser carrier frequency) in the direction of the laser wave vector. However, until this force becomes comparable to the restoring force, the majority of electrons stay as a compact dense bunch bound to the ions. When the magnitude of the laser force approaches that of the restoring force, the electrons are pushed forward so strongly that they shift considerably with respect to the ions. Immediately after this kick they turn back, pulled by the nonlinear ionic potential, cross the equilibrium point, and escape into the vacuum at the front side of the ion slab. At this instant, the electrons are accelerated orthogonally to their motion by the electric field and emit intense XUV and x-ray radiation beamed in the direction of their velocity. During the emission, the following requirements for synchrotron radiation parallel to the reflected laser axis are satisfied: (i) the electron velocity $V_{z}$ directed along the reflected wave vector is maximum in absolute value, (ii) acceleration $a_{z}$ is zero, (iii) velocity $V_{y}$ parallel to the plasma surface vanishes, and (iv) acceleration $a_{y}$ is around maximum. The time interval during which the electron velocity is orthogonal to the acceleration and parallel to the $z$ axis is confined to a small fraction of a laser cycle. Therefore, in this case the synchrotron emission, well known in physics [29] for its high intensity and high-energy spectrum, manifests itself in the form of ultrashort bursts and can form an isolated attosecond pulse, e.g., when the laser pulse consists of just a few cycles or when an ultrathin foil is used as a target [17].

This physical picture was revealed by numerical simulations with a one-dimensional PIC code modeling the interaction of a preionized plasma slab with a relativistically intense, few-cycle laser pulse of a large focal diameter $(d \gg \lambda)$. The plasma has a steep density profile with initial density $N=500 N_{c}$, where $N_{c}$ is the critical density, and $4 \mathrm{~nm}$ thickness. The plasma surface is orthogonal to the $z$ axis. The light pulse propagates along the $z$ axis from the left to the right; the linearly polarized electric field $E_{y}$ is directed along the $y$ axis and has a Gaussian envelope in time. The amplitude of the electric field is $E=$ 9.7 $\left(m c \omega_{0} / e\right)$ where $e$ and $m$ are the electron charge and mass, and the pulse's full width at a half maximum
(FWHM) is $\tau \approx 3 T_{L}$, where $T_{L}$ is the light wave period. The time and space steps are $\Delta t \approx 1.3 \times 10^{-5} T_{L}, \Delta z \approx$ $2.6 \times 10^{-5} \lambda_{0}$. Initially, 100 particles are in each mesh cell; both electrons and ions are mobile.

The characteristic times $t_{1}, t_{2}$, and $t_{3}$ of the subcycle release of plasma electrons, their acceleration, and synchrotron emission are marked by the black spheres in Fig. 1(a) showing the electric field $E_{\text {in }}$ of the incident laser pulse at the $Z$ coordinate of the front plasma surface without its modification by plasma. In the presence of the overdense plasma slab, this field changes significantly due to the interference of the incident and reflected fields. PIC simulations show that the majority of plasma electrons move together as a dense bunch until the time shortly after emission of a powerful attosecond pulse; therefore, their dynamics is well described by the motion of the maximum of the electron density together with the evolution of its spatial FWHM. The electric field $E_{N m}$ seen by the moving maximum of the electron density $N_{m}$ is shown in Fig. 1(b). This field differs from $E_{\text {in }}$ due to the plasma boundary effect and the relativistic movement of the electrons with respect to the laser pulse.

The temporal evolution of the $Z$ coordinate of $N_{m}$ is shown by the red line in the inset of Fig. 1(c) with the gray error bars depicting the spatial FWHM of the electron density. The $(Y-Z)$ trajectory of a representative electron of the emitting electron bunch obtained in PIC simulations is shown in Fig. 1(c) with the acceleration $a_{y}$ encoded in the line color. Thin black lines in Fig. 1(c) depict $(Y-Z)$ trajectories of other electrons of the emitting bunch. Note that these trajectories are plotted in the laboratory frame, not in the average rest frame, in contrast to the figure-ofeight trajectories of a single electron in relativistic fields in vacuum obtained in [30]. As is seen in the inset of Fig. 1(c), most of the electrons stay bound in the ionic potential until $t_{1} \approx 6.47 T_{L}$, when the Lorentz force overcomes the ionic binding force.

At $t_{1}$, the magnetic force has pushed the electrons far enough from the equilibrium position and their synchrotronlike motion begins. As is seen in Fig. 1(b), at the time instant $t_{2} \approx 6.79 T_{L}$, the Lorentz force at the $Z$ position of the emitting electrons recedes. Shortly after, the electrons undergo the maximum excursion in the laser direction; then they are rapidly accelerated by the restoring force of ions in the opposite direction-towards the laser. After the electron bunch passes the front surface of the ion slab, the laser field at the $Z$ position of the electron bunch provides the electric force in the $Y$ direction, thus forming, together with the ionic restoring force, a time-domain undulator. At this time instant $t_{3} \approx 7.04 T_{L},\left|V_{z}\right|=\max$, $V_{z}<0 ; V_{y}$ passes through the zero node changing sign; $a_{z}$ vanishes, while $a_{y}$ has a local maximum. As a result, a short burst of synchrotron radiation beamed in the direction of the reflected light is released. The trajectory of the maximum of the corresponding XUV and x-ray pulse 

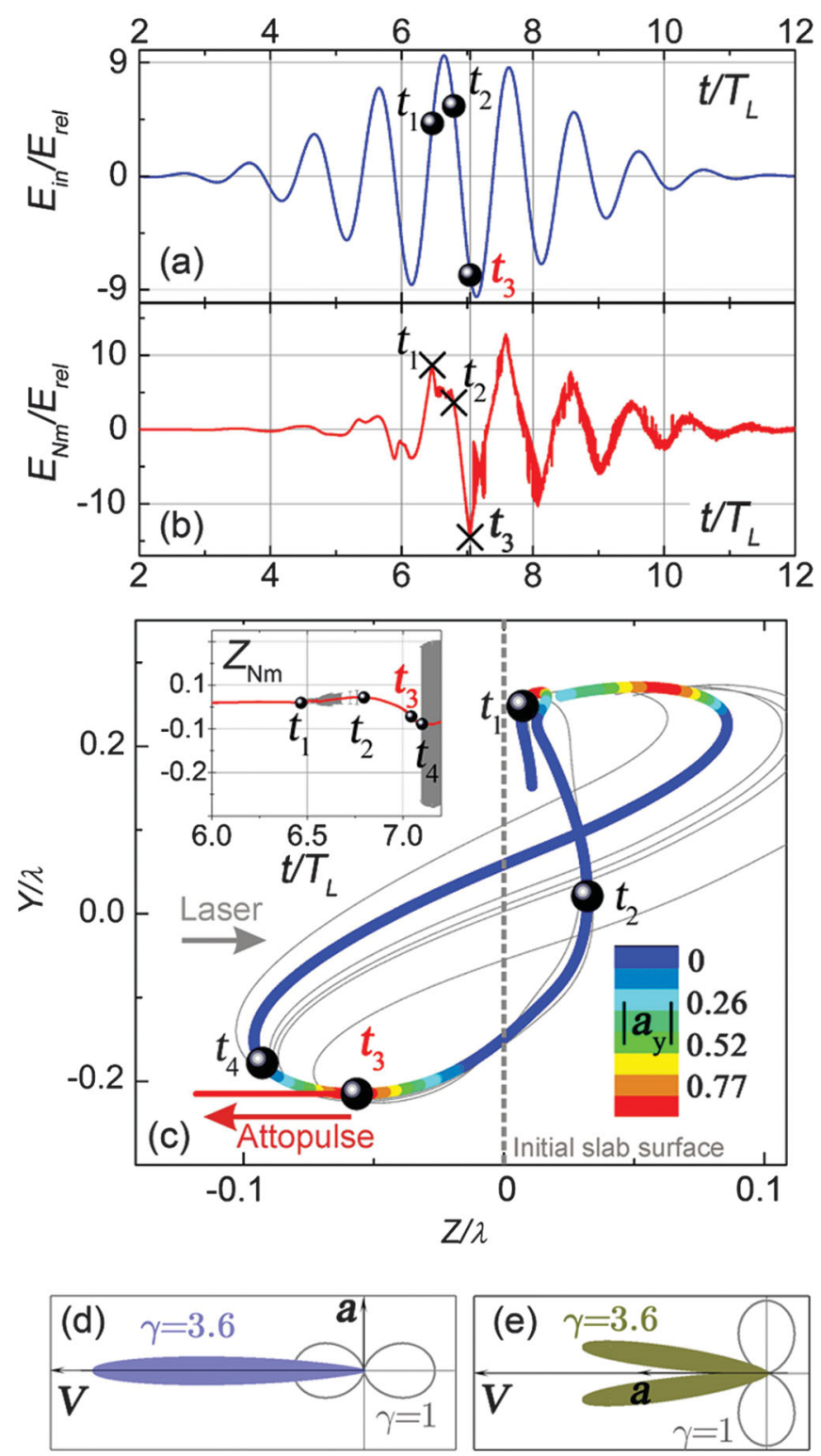

FIG. 1 (color). Clocking of a laser-driven attosecond electron synchrotron. (a) Input electric field of the driver pulse without its modification by plasma $E_{\mathrm{rel}}=m c \omega_{0} / e$. Black spheres: characteristic times of formation of the synchrotronlike electron trajectory. (b) Electric field seen by the moving maximum of the electron density with characteristic times shown by black crosses. (c) $(Y-Z)$ trajectory of a representative electron emitting synchrotron radiation (blue line) with acceleration $a_{y}$ encoded in false-color representation. Thin black lines: trajectories of other emitting electrons in the laboratory frame. Red line: trajectory of the emitted XUV and x-ray radiation. Inset: Temporal evolution of the $Z$ coordinate of $N_{m}$ (red line) with the gray error bars depicting the spatial FWHM of the electron density. (d) Radiation pattern for the synchrotronlike electron motion. The gray line depicts the pattern for $\gamma=1$, the filled pattern applies to $\gamma=3.6$ and is reduced by a factor $\sim 10^{5}$ for the same acceleration. (e) Radiation pattern for an electron accelerated in its direction of motion. The gray line depicts the pattern for $\gamma=1$; the filled pattern applies to $\gamma=3.6$ and is reduced by a factor $\sim 10^{4}$ for the same acceleration. obtained in PIC simulations is shown by the red line in Fig. 1(c).

The trajectories of most of the electrons coincide [black lines in Fig. 1(c) merge with the blue line] until $t_{4} \approx 7.1 T_{L}$, when the acceleration $a_{y}$ vanishes. Following this, once the bunch has been decelerated, it explodes due to Coulomb repulsion. Electrons start moving incoherently [the lines in Fig. 1(c) split, and the error bars in the inset of Fig. 1(c) become large at $t>t_{4}$ ]. Therefore, they cannot produce a second intense attosecond pulse during the subsequent half-cycle of the laser driver field.

The radiation pattern of a single electron accelerated orthogonally to its direction of motion [29] is shown in Fig. 1(d) juxtaposed with that of an electron accelerated in the direction of its motion, Fig. 1(e). In the latter case, for the same magnitude of applied force the radiation is a factor of $\gamma^{2}$ smaller than that with a transverse acceleration, and vanishes in the direction of an electron velocity. The fact that in PIC simulations and experiment [19-21] the maximum of the XUV emission propagates along the $Z$ axis corroborates its "synchrotron" origin whose pattern is given by Fig. 1(d). Note that this radiation pattern refers to a single electron. If electrons are driven coherently by a spatially coherent incident wave, many of them move along the same trajectory, resulting in a coherent reflected wave in a diffraction-limited beam whose characteristics are dictated by the coherence properties of the driving field rather than by the single-electron emission properties.

The velocity and acceleration of tens of selected plasma electrons contributing to the attosecond pulse emission versus time are shown in Figs. 2(a) and 2(b). The selected electrons represent the majority of electrons of the slab. As is seen, electrons move "coherently" (the curves for different electrons coincide) until $t_{4}$. Therefore, the synchrotronlike behavior at around $t_{3}$ is also reproduced in the macroscopic currents $J_{y}$ and $J_{z}$ demonstrated by Fig. S3 of the Supplemental Material [28]. The reflected field and its intensity after spectral filtering are depicted in Fig. 2(c), where the isolated attosecond pulse is clearly observed.

Figures 2(d) and 2(e) demonstrate $V_{y}, V_{z}, a_{y}$, and $a_{z}$ of a representative electron of the emitting bunch on a zoomedin time scale near $t_{3}$. The intensity of the generated attosecond pulse and its $Z$ coordinate are shown by the red line; the dashed line shows the $Z$ coordinate of the electron. At the instant $t_{3}$, initially bound electrons have become quasifree, or "highly excited" in the language of atomic physics. The trajectories of these electrons obtained in selfconsistent PIC simulations are similar to those of a single electron in a relativistically intense electromagnetic pulse in vacuum [31], but with essential differences in the minima of $V_{z}$, as shown in the Supplemental Material [28].

An analytical expression for the intensity of the attosecond pulse can be deduced from the electron trajectories shown in Figs. 1, 3, and 3 by using approximations to $V_{z}$ 


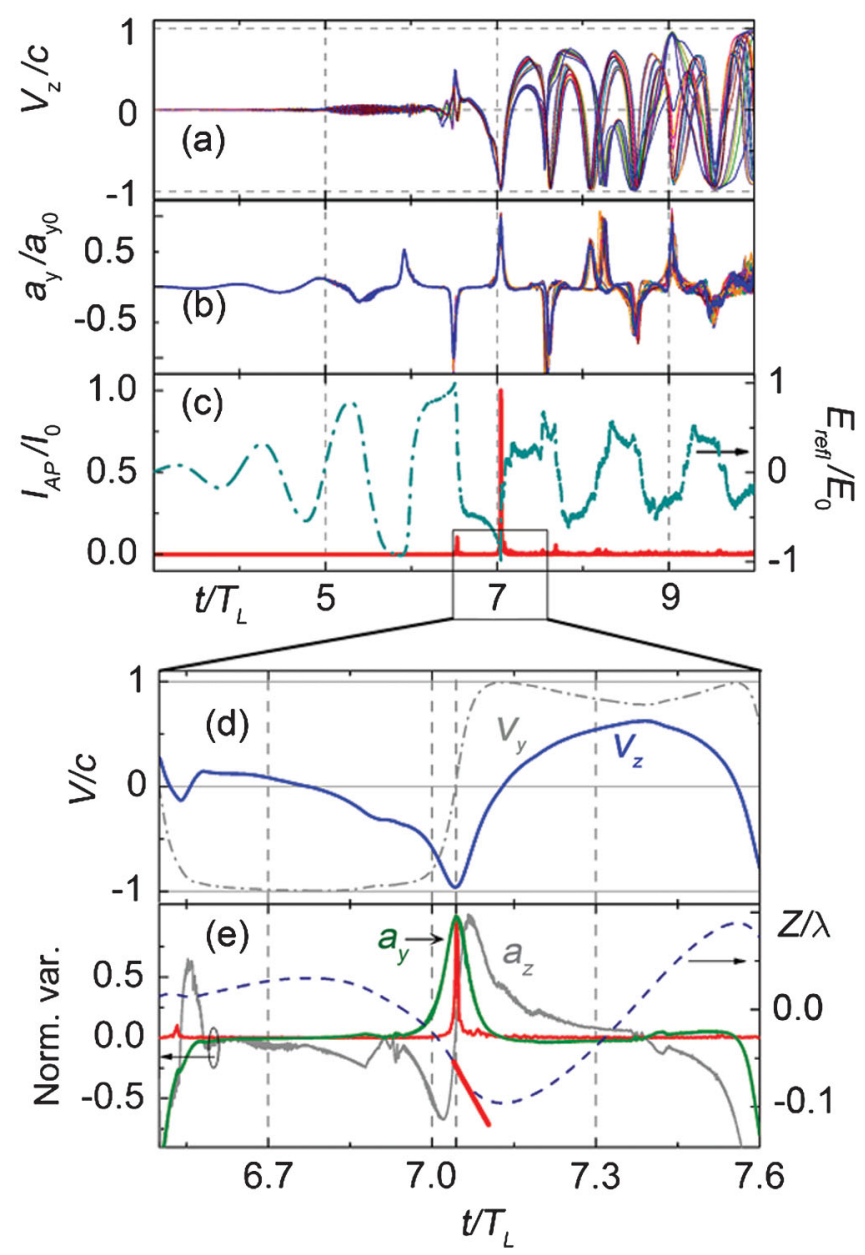

FIG. 2 (color). Dynamics of emitting electrons obtained with PIC simulations. (a), (b) $V_{z} / c$ and $a_{y}$ (normalized to their maxima $a_{y 0}$ at $t_{3}$ ) of a number of different electrons as a function of time. (c) Reflected electric field (cyan line) and its intensity $|E(t)|^{2}$ after spectral filtering $\omega>80 \omega_{0}$ (red line) normalized to their maxima. (d), (e) $V_{y} / c$ (gray dash-dotted line), $V_{z} / c$ (blue solid line), $a_{y} / a_{y 0}$ (dark green line), and $a_{z} / a_{z 0}$ (gray line), as well as the coordinate $Z / \lambda$ (dashed line) of a representative electron near the emission time $t_{3}$. Red line: intensity and $Z$ coordinate of the attosecond pulse.

and $a_{y}$ obtained in PIC simulations, because a sufficient number of electron trajectories coincide during the attosecond pulse emission. The acceleration $a_{y}(t)$ shown in Fig. 3 can to a good approximation be written as $a_{y}(t)=$ $a_{y 0} \exp \left[-\left(t / \tau_{a}\right)^{2}\right]$, while the velocity $V_{z}(t)$ can be represented near its maximum by a parabolic function

$$
V_{z}(t) \approx V_{z 0}\left(1-\frac{\alpha t^{2}}{2}\right) \approx c\left(1-\frac{1}{2 \gamma^{2}}-\frac{\alpha t^{2}}{2}\right)
$$

where $\alpha=d^{2} V_{z} / d t^{2}$. The functions $V_{z}(t)$ and $a_{y}(t)$ determine both the duration and the intensity of the attosecond pulse. Using the framework of Liénard-Wiechert potentials, the electric field radiated by the accelerated electrons

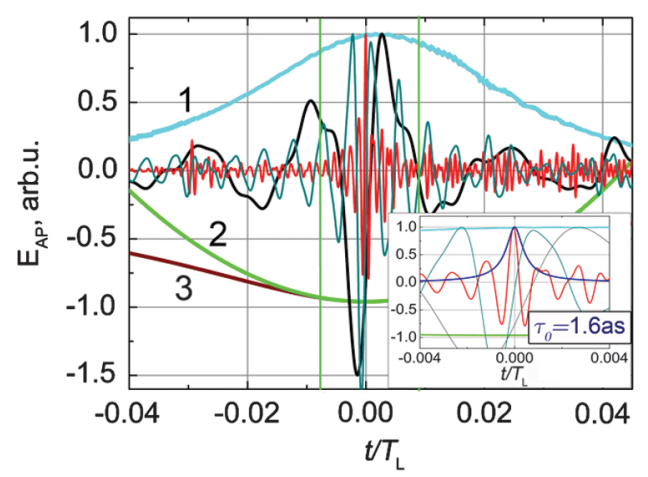

FIG. 3 (color). Attosecond pulses obtained by applying three different spectral filters to the reflected field from Fig. 2(c). Black line: (50-200 $\left.\omega_{0}\right)$, dark cyan: $\left(200-600 \omega_{0}\right)$, red: $\left(700-1500 \omega_{0}\right)$. Line 1: normalized $a_{y}(t)$; line 2: $V_{z}(t)$; line 3: the approximation Eq. (1); green vertical lines show the interval of validity of Eq. (1). Inset: same plots on a zoomed-in time axis. Dark blue line: electric field given by Eq. (2).

can be expressed as (see the Supplemental Material [28] for derivation)

$$
E(t)=-\frac{N e}{c^{2} R} a_{y}\left(t^{\prime}\right) \frac{4 \gamma^{4}}{\left(1+\alpha \gamma^{2} t^{\prime 2}\right)^{2}},
$$

where $N$ is the full number of electrons moving coherently near the time of emission, and the retarded time $t^{\prime}(t)$ is given by

$$
t^{\prime}=\frac{2 \gamma^{2}(t-z / c)}{\sqrt{1+\left[(t-z / c) \nu_{0}\right]^{4 / 3}}},
$$

where $\nu_{0}=\gamma^{3} \sqrt{\alpha} /(\sqrt{3} / 2)$.

Here, we have introduced the key scaling parameter $\nu_{0} \sim \gamma^{3} \sqrt{\alpha}$, which defines the high-frequency cutoff of the radiation. The $\gamma^{3}$ factor reveals a striking similarity with the spectrum of synchrotron radiation [29]. It is straightforward to see from Eq. (3) that the fast growth of the nonlinear function $t^{\prime}(t)$ is confined to the time interval $\tau_{0} \sim\left(\gamma^{3} \sqrt{\alpha}\right)^{-1}$ (see Fig. S5 of the Supplemental Material [28]). Electrons emit high-intensity radiation only as long as the velocity $V_{z}$ is high in its absolute value and $t^{\prime}(t)$ is undergoing a rapid nonlinear growth within the characteristic time $\tau_{0}$. In other words, $\tau_{0}$ provides a good estimate for the pulse width of ultrashort XUV and X-ray bursts emitted by the electrons.

Attosecond pulses obtained by applying three different spectral filters to the reflected light field obtained in PIC simulations are demonstrated in Fig. 3. With an incident laser intensity of $I_{0}=1.8 \times 10^{20} \mathrm{~W} / \mathrm{cm}^{2}$ and central wavelength of $810 \mathrm{~nm}$, the intensity of the attosecond pulse is $I \approx 3 \times 10^{18} \mathrm{~W} / \mathrm{cm}^{2}$ for the spectral interval from $50 \omega_{0}$ to $200 \omega_{0}$ (duration 52 as); $I \approx 4 \times 10^{17} \mathrm{~W} / \mathrm{cm}^{2}$ from $200 \omega_{0}$ to $600 \omega_{0}$, (duration 15 as); $I \approx 2 \times 10^{16} \mathrm{~W} / \mathrm{cm}^{2}$ from $700 \omega_{0}$ to $1500 \omega_{0}$ (duration 1.6 as). The inset of 
Fig. 3 shows same plots on a zoomed-in time scale, where the dark blue line depicts the electric field given by Eq. (2). The shortest duration of the emitted XUV and x-ray pulse is $\tau_{0}=5.9 \times 10^{-4} \cdot T_{L} \approx 1.6 \mathrm{as}$. Green vertical lines in Fig. 3 show the interval of validity of the parabolic approximation to $V_{z}$.

In summary, we have derived an analytical formula describing the electric field of attosecond pulses emitted from a laser-produced overdense plasma slab as a function of time, based on the trajectories of relativistic electrons obtained with PIC simulations, which can be generalized to other configurations of laser-solid interaction including oblique incidence, thick targets, and nonplanar geometries. Using analytical expressions for the electron acceleration and velocity as a function of laser and plasma parameters, one can deduce the scaling of the attosecond pulse intensity and duration as a function of experimentally measurable parameters. Our new interpretation of the emission of relativistic harmonics from solids, where the entire system-the overdense plasma in concert with the laser field-momentarily forms a synchrotron source, active at a certain instant of time and governed by a simple set of rules, will assist the development and optimization of sources of intense attosecond pulses.

We are grateful for support by the Max-Planck Society and the Cluster of Excellence: Munich Center for Advanced Photonics (MAP). The work is partially supported by the Russian Foundation for Basic Research Grants No. 11-02-01043 and No. 11-02-01217, as well as Skolkovo Foundation Grant No. 78. J. M. M. and N. K. gratefully acknowledge support by the Alexander-vonHumboldt Foundation. A. M.Z. gratefully acknowledges a partial support of his research by the Welch Foundation Grant No. A-1801. P. G. acknowledges support through the Helmholtz Alliance HA216/EMMI. J. M. M. is grateful to N. A. Tolstikhina and M. V. Mikhailov.

*julia.mikhailova@mpq.mpg.de

[1] G. A. Mourou and T. Tajima, Science 331, 41 (2011).

[2] M. Hentschel, R. Kienberger, Ch. Spielmann, G. A. Reider, N. Milosevic, T. Brabec, P. Corkum, U. Heinzmann, M. Drescher, and F. Krausz, Nature (London) 414, 509 (2001).

[3] A. Baltuska et al., Nature (London) 421, 611 (2003).

[4] G. Sansone et al., Science 314, 443 (2006).
[5] E. Goulielmakis et al., Science 320, 1614 (2008).

[6] P. B. Corkum, Phys. Rev. Lett. 71, 1994 (1993).

[7] A. McPherson, G. Gibson, H. Jara, U. Johann, T. S. Luk, I. A. McIntyre, K. Boyer, and C. K. Rhodes, J. Opt. Soc. Am. B 4, 595 (1987).

[8] X.F. Li, A. L'Huillier, M. Ferray, L. A. Lompré, and G. Mainfray, Phys. Rev. A 39, 5751 (1989).

[9] M. Uiberacker et al., Nature (London) 446, 627 (2007).

[10] E. Goulielmakis et al., Nature (London) 466, 739 (2010).

[11] A. L. Cavalieri et al., Nature (London) 449, 1029 (2007).

[12] F. Krausz and M. Ivanov, Rev. Mod. Phys. 81, 163 (2009).

[13] S. V. Bulanov, N. M. Naumova, and F. Pegoraro, Phys. Plasmas 1, 745 (1994).

[14] P. Gibbon, Phys. Rev. Lett. 76, 50 (1996).

[15] R. Lichters, J. Meyer-ter-Vehn, and A. Pukhov, Phys. Plasmas 3, 3425 (1996).

[16] L. Plaja, L. Roso, K. Rzazewski, and M. Lewenstein, J. Opt. Soc. Am. B 15, 1904 (1998).

[17] Yu. M. Mikhailova, V.T. Platonenko, and S.G. Rykovanov, JETP Lett. 81, 571 (2005).

[18] T. Baeva, S. Gordienko, and A. Pukhov, Phys. Rev. E 74, 046404 (2006).

[19] B. Dromey et al., Nat. Phys. 2, 456 (2006).

[20] G. Mourou, T. Tajima, and S. V. Bulanov, Rev. Mod. Phys. 78, 309 (2006).

[21] A. Tarasevitch, K. Lobov, C. Wünsche, and D. von der Linde, Phys. Rev. Lett. 98, 103902 (2007).

[22] F. Quéré, C. Thaury, P. Monot, S. Dobosz, Ph. Martin, J.-P. Geindre, and P. Audebert, Phys. Rev. Lett. 96, 125004 (2006).

[23] J. M. Mikhailova, in Proceedings of the MPQ Seminar, Garching, Germany, 2009 (unpublished); in Proceedings of the JSC Seminar, Juelich, Germany, 2009 (unpublished).

[24] D. an der Bruegge and A. Pukhov, Phys. Plasmas 17, 033110 (2010).

[25] H. Alfvén and N. Herlofson, Phys. Rev. 78, 616 (1950).

[26] A. G. Pacholczyk, Radio Astrophysics (Freeman, San Francisco, 1970).

[27] F. R. Elder, A. M. Gurewitsch, R. V. Langmuir, and H. C. Pollock, Phys. Rev. 71, 829 (1947).

[28] See Supplemental Material at http://link.aps.org/ supplemental/10.1103/PhysRevLett.109.245005 for additional PIC simulation results and derivations.

[29] J. D. Jackson, Classical Electrodynamics (John Wiley \& Sons, New York, 1962).

[30] E. S. Sarachik and G. T. Schappert, Phys. Rev. D 1, 2738 (1970).

[31] L. D. Landau and E.M. Lifshitz, Classical Theory of Fields (Addison-Wesley, Reading, MA, 1962), 2nd ed. 